\title{
Skin Transplantation and Lymphoid Organ Analysis in Mice
}

Julie Ruer-Laventie ${ }^{1}$, Rajesh Jayachandran ${ }^{1}$, Mathias Schmaler², Simona W. Rossi ${ }^{2, *}$ and Jean Pieters ${ }^{1, *}$

${ }^{1}$ Biozentrum, University of Basel, Basel, Switzerland; ${ }^{2}$ Department of Biomedicine, University and University Hospital Basel, Basel, Switzerland

*For correspondence: iean.pieters@unibas.ch; simona.rossi@unibas.ch

\begin{abstract}
[Abstract] Skin transplantation in mice is an important procedure to evaluate immune responses generated against heterologous grafts, especially given its highly immunogenic nature. In fact, skin is one of the most challenging organs in terms of allograft retention. In this protocol, we provide a detailed procedure for skin grafting using the tail skin as donor organ that is grafted on the dorsal site of thoracic cage in a recipient mouse. We also provide protocols for the systematic analysis of lymphoid organ analysis in transplanted mice. Together these protocols may be valuable for evaluation of parameters that affect skin grafting, including genetic factors, immune cell activation as well as the analysis of compounds that may be useful in allowing graft tolerance.
\end{abstract}

Keywords: Skin transplantation, Mouse, Lymphoid organs, Immune cell analysis, Protocol, Organ rejection

[Background] Transplantation of organs remains the only life-saving procedure for many end-stage organ failures. However, the success of organ transplantation is severely limited by first, the availability of a suitable donor with a matched Human Leucocyte Antigen (also known as HLA typing/organ matching), and second, the fact that even in cases of matched transplants, recipients have to be subjected to immunosuppressive therapy where multiple drugs are administered life-long, resulting in severe side effects (Lopez et al., 2006; Green, 2013; Rodriguez-Peralvarez et al., 2014). Therefore, a better understanding of the factors that allow graft acceptance, as well as the availability of sensitive protocols to assay immune cell activation are important. We have employed a mouse model for skin transplantation, where a biopsy from a tail skin is grafted onto the dorsum of a recipient mouse (Schmaler et al., 2014; Jayachandran et al., 2019). Such a procedure not only allows for relative ease of obtaining tail skin graft from a single donor for performing transplantation onto multiple recipients, but also aids in rapid assessment of the graft health status due to the presence of the characteristic hyperpigmented tail stripe (from melanin, a characteristic feature of healthy tail skin), that rapidly fades upon rejection, thereby allowing a relatively straightforward evaluation of its tolerance and/or rejection following transplantation. 


\section{Materials and Reagents}

A. For the skin transplantation protocol

1. Mouth mask (Lyreco, catalog number: 5965655)

2. Cotton swab (Carl Roth $\mathrm{GmbH}$, catalog number: 31025060)

3. Hansaplast Finger Strips (Beierdorf AG, catalog number: 76861)

4. Histoacryl Tissue adhesive (Braun, catalog number: 1050052)

5. Leukotape classic, $2 \mathrm{~cm} \times 10 \mathrm{~m}$ (BSN Medical SAS, Ref: 02204-00)

6. Sterile gauze, $5 \times 5 \mathrm{~cm}$, 8 ply (MaiMed $\mathrm{GmbH}$, catalog number: 21010)

7. Tissue Culture dishes $10 \mathrm{~cm}, 60.1 \mathrm{~cm}^{2}$ (Huberlab, catalog number: 7.633102 )

8. Warm pad (Solis AG, Type 223)

9. $1 \mathrm{ml}$ syringes with $25 \mathrm{G}$ needles or insulin syringes (BD, catalog number: 305501 )

10. Heating pad (surgery table with a heating block included; MD supplies, catalog number: 45071/)

11. Cotton swabs (Schaffhauser, catalog number: P0-15117601)

12. Mice (H2-bm12) (H-2 ${ }^{\mathrm{bm} 12}$, McKenzie et al., 1979) and C57BI/6

13. $\mathrm{CO}_{2}$ for euthanization

14. Wet food ( $100 \mathrm{~g}$ of dry food incubated at room temperature with $200 \mathrm{ml}$ of drinking water $\mathrm{O} / \mathrm{N}$, $30 \mathrm{ml}$ of mix is placed on a Petri dish per cage) (KLIBA, 3436 EXF12 M/R EXTRUDAT)

15. Betadine standard solution (Mundipharma)

16. Dafalgan (UPSA, Bristol Myers Squibb SA, 1340235)

17. PBS, Phosphate-Buffered Saline, pH 7.4 (Invitrogen, catalog number: 10010015)

18. Temgesic, Buprenorphine, $0.3 \mathrm{mg} / \mathrm{ml}$ (ESSEX Chemie AG, ATC N02AE01 number)

19. Vaseline (Vifor SA)

20. Isoflurane (Attane, SKU:RXISO-250)

21. Vitamin A (Blache, catalog number: 1667290), $5 \mathrm{~g}$, Bausch \& Lomb for the vet ointment of the mice eyes

22. $100 \%$ Ethanol, vendor (Sigma, catalog number: 02890)

23. Foetal Calfs Serum (FCS) (PAA, catalog number: A15-101)

24. Paracetamol-containing syrup (Bristol-Myers Squibb, DAFALGAN Sirup, $30 \mathrm{mg} / \mathrm{ml}$ )

B. For the organ analysis

1. $1 \mathrm{ml}$ syringes with $25 \mathrm{G}$ needles or insulin syringes (BD, catalog number: 305501 )

2. Gridded mesh (steel/nylon, Sefar AG, catalog number: 3053-0201-100-00)

3. Neubauer chamber (BRAND, catalog number: 718605)

4. Petri dishes $94 \times 16 \mathrm{~mm}$ (Greiner, catalog number: 633102)

5. $15 \mathrm{ml}$ tubes (Sarstedt, catalog number: 62.554.502)

6. $5 \mathrm{ml}$ plungers (Omnifix, catalog number: $4616057 \mathrm{~V}$ )

7. $5 \mathrm{ml}$ facs tubes (Falcon, catalog number: 352008 ) 
8. 96-well plate U bottom non-treated for culture (VWR, 351177 Falcon, 734-0955)

9. Live/Dead Marker (Invitrogen, catalog number: L10119)

10. $2 \%$ EDTA (Sigma, EDS-100g)

11. ACK buffer ( $155 \mathrm{mM} \mathrm{NH}_{4} \mathrm{Cl}, 10 \mathrm{mM} \mathrm{KHCO}_{2}, 1 \mathrm{mM}$ EDTA, $\mathrm{pH}$ 7.4) to use for RBC lysis in organs

12. Aqua dest. 10x BD FACS Lysing Solution (BD Biosciences, catalog number: 349202) (dilute with aqua dest) to use for RBC in blood

13. FACS buffer (PBS, $2 \%$ FCS, 5 mM EDTA)

14. Trypan blue stain (0.4\%, Gibco, catalog number: $15250-061)$

15. FACS antibodies list example of panel which can be used:

CD3 (Clone 145-2C11, Biolegend, catalog number: 100308)

CD4 (Clone RM4-5, Biolegend, catalog number: 100512)

CD8 (Clone 53-6.7, Biolegend, catalog number: 100738)

CD19 (Clone 6D5, Biolegend, catalog number: 115508)

Gr1 (Clone RB6-8C5, Biolegend, catalog number: 108425)

CD62L (Clone Mel-14, Biolegend, catalog number: 104412)

CD44 (Clone A20, Biolegend, catalog number: 103030)

CD11b (Clone M1/70, Biolegend, catalog number: 101236)

CD80 (Clone 16-10A1, Biolegend, catalog number: 104702)

CD86 (Clone GL-1, Biolegend, catalog number: 105010)

\section{Equipment}

A. Surgical instruments for the skin transplantation protocol

1. Narrow pattern forceps (FST, catalog number: 11003-12)

2. Fine iris scissor curved (FST, catalog number: 14095-11)

3. Fine iris scissor (FST, catalog number: 14094-11)

4. Mayo scissors (FST, catalog number: 14010-15)

5. Artery scissors ball tip $11.5 \mathrm{~cm}$ (FST, catalog number: 14080-11)

6. Tissue forceps (FST, catalog number: 11021-14)

7. Carbon Steel, Surgical Blade No.20 (Swann-Morton LTD, catalog number: 3006)

8. Surgical blade Handles (Swann-Morton LTD 20, Milian, catalog number: 132620SW)

B. For the skin transplantation protocol

1. Electric shaver (Moser Classic A, Barthaar, catalog number: 1040.0460)

2. Ear tweezer (FST, catalog number: 24212-01)

C. For the organ analysis

1. BD FACS Canto II cytometer, IC/NO: 0000031701

2. Vacuum pump (KNF Neuberger, catalog number:N816.3KN.18) 


\section{Software}

1. FlowJo software (LLC, BD (Becton, Dickinson \& Company, https://www.flowjo.com/)

2. BD FACSDiva Software (version8.0.1, firmware version 1.49 BD FACSCanto II)

\section{Procedure}

Note: For all procedures approval should be obtained from the appropriate animal use committees.

\section{Part I: Tail-skin transplantation}

Note: Perform all procedures as sterilely as possible. References: Schmaler et al. (2014); Jayachandran et al. (2019).

\section{A. Preparation of surgery}

All instruments and materials (surgical equipments, cotton swab, gauze), should be autoclaved.

1. Prepare and organize instruments and materials as mentioned in Materials and Reagents section A1-A22 and Equipment section A1-A8, B1-B2 as well as turn on the anesthesia mobil unit (Figure $1 \mathrm{~A}$ ) and warm up the heating pad (Figure 1B).

2. Open the finger strip bandages and apply Vaseline on the wound pad (use a cotton swab ensuring the gauze is covered) (Figure 1C).

3. Prepare 10-15 cm strips of leukotape per mouse (Figure 1D).

4. Administer analgesia: Buprenorphine $0.05 \mathrm{mg} / \mathrm{kg}$ body weight is administered intraperitoneally using an insulin syringe post dilution in PBS in a volume of $100 \mu \mathrm{l}$ (weigh the mouse!) to ensure analgesia immediately after the surgery.
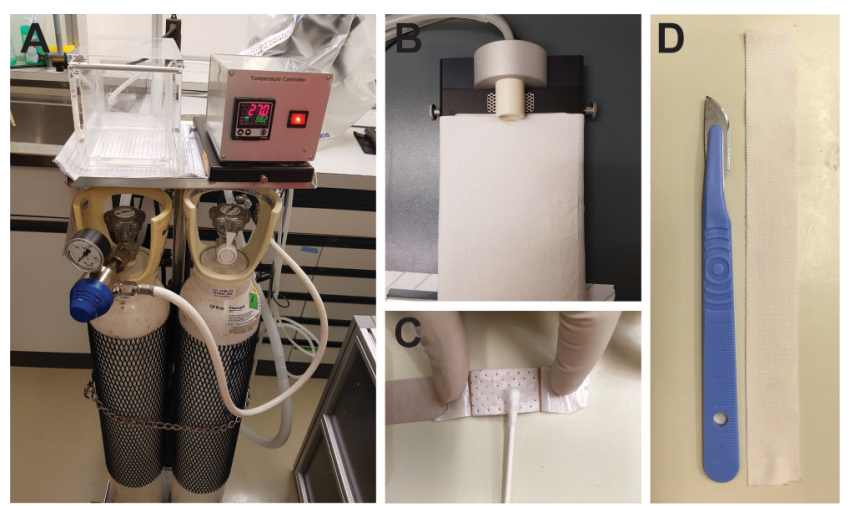

Figure 1. Preparation of the material for the surgery. The surgical set-up prepared for skin transplant surgery: anesthesia mobile unit (A) and warm pad (B). The Hansaplast plaster is coated with Vaseline on the non-adhesive area with a cotton swabs (C). $10-15 \mathrm{~cm}$ of leukotape strips are prepared (D). 
B. Preparation of the tail-skin for transplantation

1. Donor mice [e.g., $\mathrm{H} 2-\mathrm{bm} 12\left(\mathrm{H}-2^{\mathrm{bm} 12}\right)$ (McKenzie et al., 1979)] are euthanized by $\mathrm{CO}_{2}$ asphyxiation.

2. Disinfect the tails with $70 \%$ ethanol.

3. Place the animal on a surgical board with its tail black stripe facing down. Dissect the tail longitudinally throughout the mid-line (here marked with a marker to make it easier to see Figure $2 A)$.

4. Make a circular incision at the proximal end of the tail and a superficial incision only involving the skin layer starting from the proximal to the distal end.

5. Use a tissue forceps to peel off the tail skin starting from the proximal site (where the circular incision is seen (Figure 2B) to the distal end (Figure 2C) and transfer the tail skin into a $10 \mathrm{~cm}$ Petri dish with $10 \mathrm{ml}$ of PBS (Figure 2D).

6. Using a scalpel, make a transplant to match the size of the incision done on the dorsal part of the recipient (see Figure3), make a round/circle using the scalpel (Figures 2E-2G).
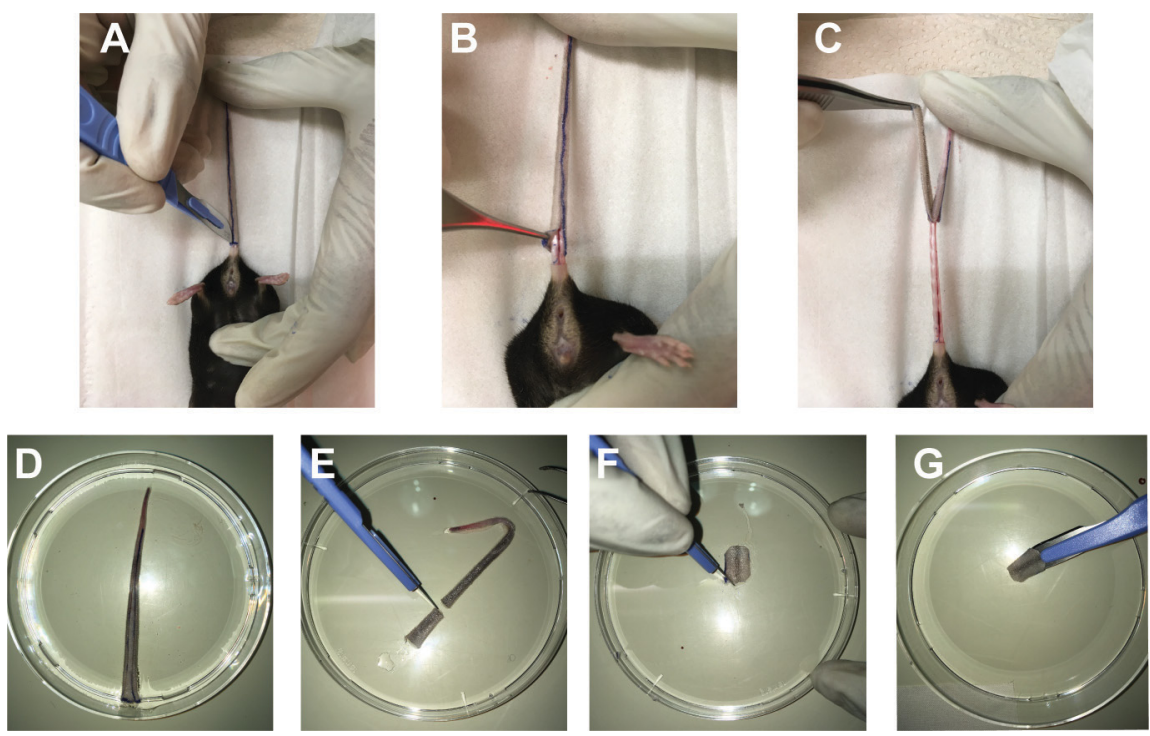

Figure 2. Donor tail skin graft preparation. A. The tail donor is dissected from the proximal to the distal end with a scalpel. The skin of the tail is pulled off from the proximal (B) to the distal end (C). D. The donor tail skin graft is stored in sterile PBS. A piece of the graft is dissected using a scalpel $(E)$ and reshaped on the corners $(F)$ then placed on the scalpel blade for placement in to graft bed prepared on the recipients' dorsal skin $(G)$.

\section{Surgical Procedure}

1. The recipient mouse (e.g., $\mathrm{C} 57 \mathrm{BL} / 6, \mathrm{H}-2^{\mathrm{b}}$ ) is anesthetized by inhalation of a $3 \%$ Isofluorane solution (it should take 3-5 min to reach surgical plane anesthesia) in a closed chamber. Once anesthetized, vitamin A ointment is applied to the eyes to prevent corneal dryness while under anesthesia. 


\section{b̆̈o-protocol}

2. Transfer the mouse to the cushioned warm pad maintained at $37^{\circ} \mathrm{C}$ and apply $1.5 \%$ Isofluorane via a mouth mask (check the reflexes by toe pinching to make sure the mouse is properly anesthetized).

3. The dorsal site of the recipient mouse skin (e.g., C57BL/6), about $2 \mathrm{~cm}$ below the ear line is shaved $\left(3 \mathrm{~cm}^{2}\right.$ area) and any hairs removed using a dry gauze swab (Figure $3 \mathrm{~A}$ ).

4. Use betadine solution to disinfect the transplantation site using a cotton swab (Figure 3B).

5. Lift the skin upwards using forceps in the middle/right site of the dorsum and make a round incision using curved scissors $(\sim 1 \mathrm{~cm} \times 0.6 \mathrm{~cm})$ (Figure $3 \mathrm{C}$ ).

6. Immediately clean the wound when bleeding occurs (using a sterile cotton swab).

7. Dry off any excess PBS using a sterile cotton swab and place the transplant onto the recipient mouse (use a scalpel) (Figure 3E).

8. Use tissue adhesive (Histoacryl) to fix the transplant (only on the contact zone) to the recipient skin avoiding any overlap of donor and host skin (Figure 3F).
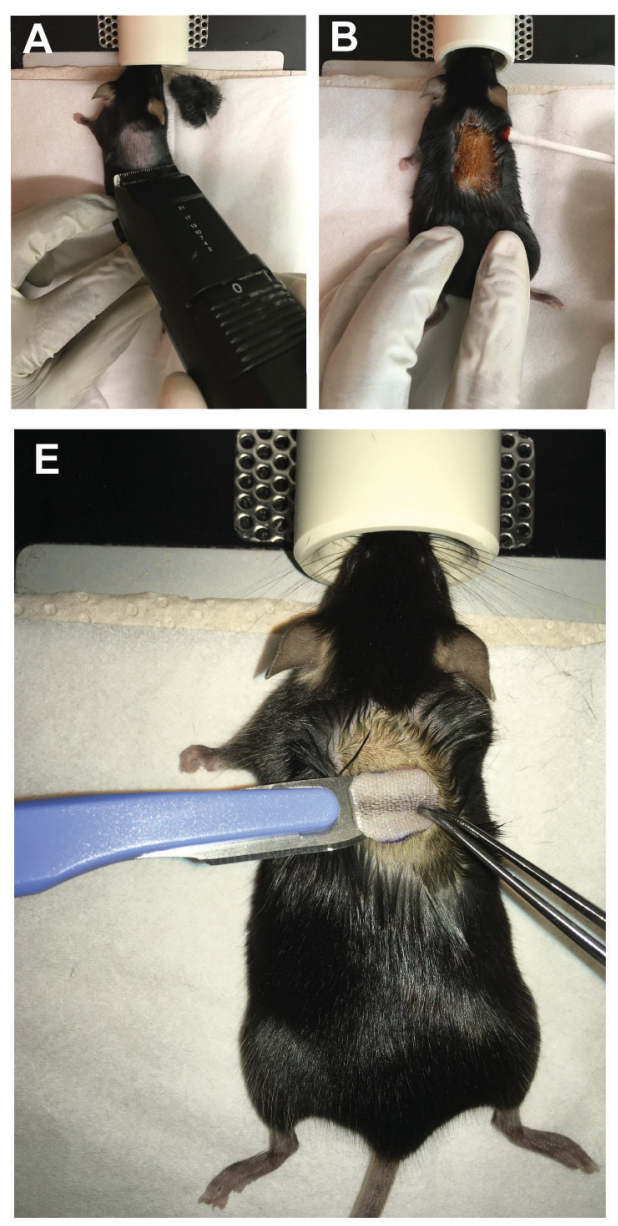
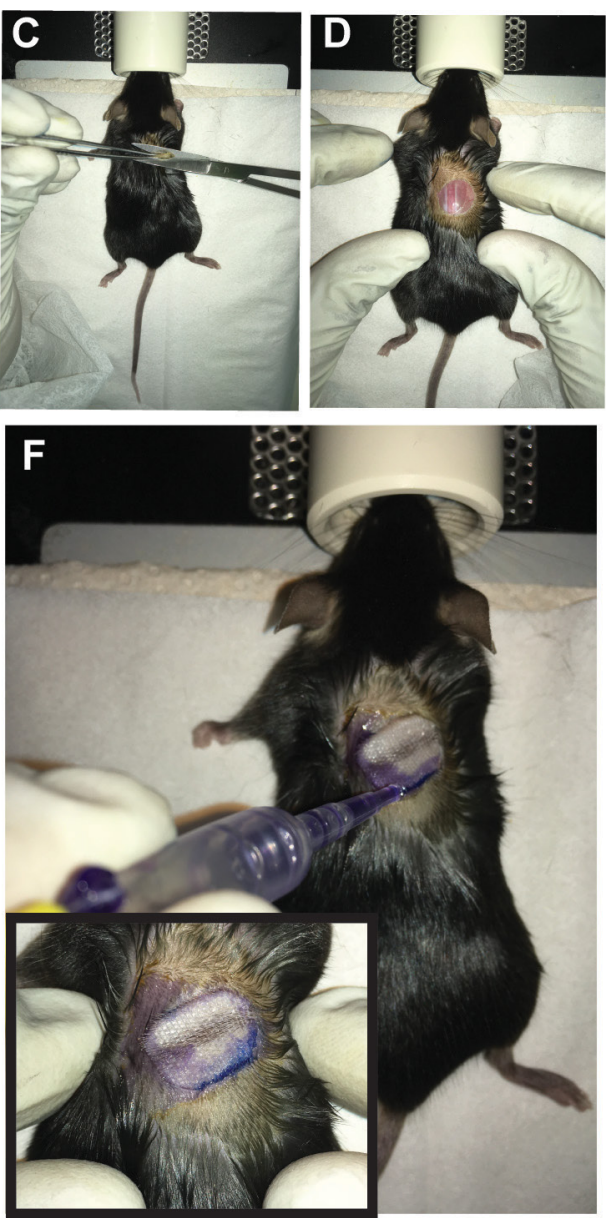

Figure 3. Preparation of the dorsal part of the recipient mouse for surgical implantation

of the donor tail skin. The recipient mouse is placed under the anesthetic mask and its dorsal skin is shaven (A) and cleaned with Betadine (B). The dorsal skin is grasped with a blunt end forceps and a small area of skin about $1 \mathrm{~cm}^{2}$ is snipped of with a curved scissor (C). The graft bed is prepared to host the recipients' dorsal skin (D). The donor tail graft is gently placed $(E)$ 
within the surgical opening created on the dorsal surface of the recipient and glued with the surrounding dorsal skin using Histoacryl without any overlapping on recipient's skin (F).

D. Protection of the graft after surgery

1. Once the Histoacryl dries up (1-2 min), cover up the surgical site with a plaster. Carefully wrap a plaster (Hansaplast Finger Strips, no creases) around the mouse waist (see Figures 4A-4B).

2. Fix the plaster using an adhesive non-elastic bandage to the mouse $(3 \mathrm{~mm}$ shifted posteriorly relative to the anterior margin of the Hansaplast plaster) (Figures 4C-4D).

3. To prevent the mouse's teeth to be trapped during the recovery phase, make a $\sim 3 \mathrm{~mm}$ incision in the upper ventral site of the bandage (Figure 4E).

4. When fully recovered the transplanted mice are transferred to their cages and mobility is assessed (Figure 4F) (bandage should not be too tight; if the bandage is too loose or too tight, remove the old bandage (under anesthesia) and reapply the bandage). At this point, the animals receive wet food until bandage removal.

5. Sterilize all instruments $(70 \%$ ethanol) prior to performing the subsequent surgery.
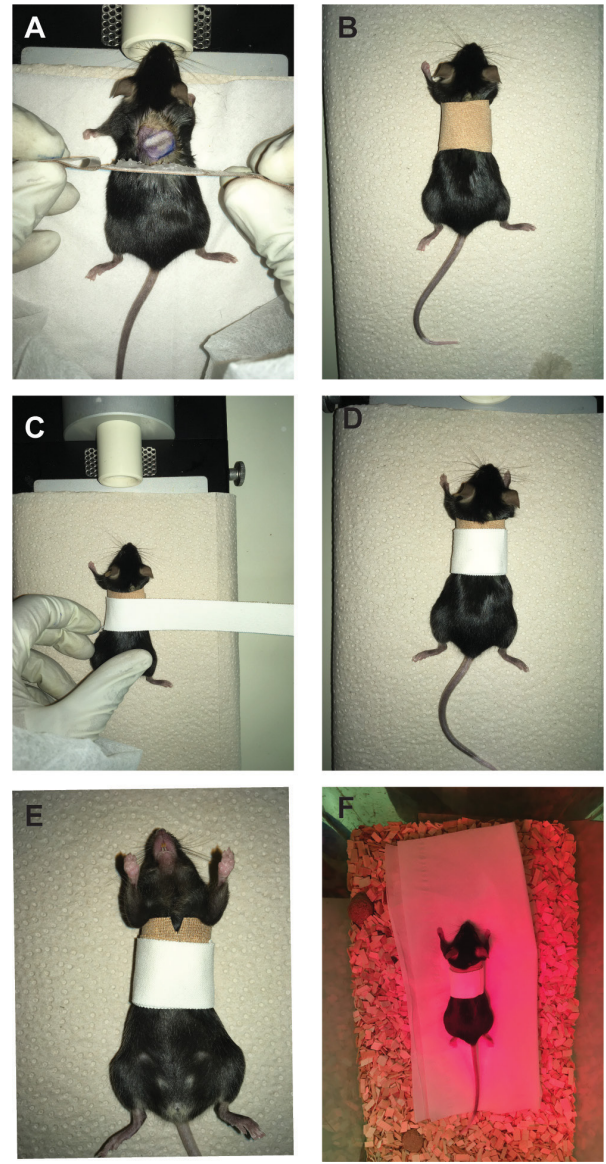

Figure 4. Protection of the graft after surgery. For plaster application, a Hansaplast plaster that has been generously coated with Vaseline on the non-adhesive area is gently layered on top of the graft and rounded up along the girth of the abdomen (A-B). Subsequently, another 
layer of Leukotape (white in the figure) bandage is applied to firmly fix the graft in place (C-D). A $3 \mathrm{~mm}$ incision in the upper ventral site of the bandage (E). The mouse is placed back in a transitional cage under a red lamp to warm up and allowed to wake up $(F)$.

E. Post-operative Care

1. Analgesia is provided by using paracetamol-containing syrup in the drinking water $(4 \mathrm{mg} / \mathrm{ml})$ for 7 days.

2. Once fully recovered, as judged by alertness and mobility, the mouse can be returned to the animal room. This takes usually $\sim 30 \mathrm{~min}$; Housing can be in either single or grouped cages.

3. Transplanted mice should be monitored for behavioral changes (eating or immobility), abnormal appearance (lack of grooming) or posture (pilo-erection or hunched posture) for the time the bandage is in place (1 week). If animals are unable to move (e.g., trapped bandage), re-apply the bandage under anesthesia.

F. Bandage removal (6-7 days post-transplantation)

1. Initiate anesthetizing the mouse through inhalation of a $3 \%$ Isofluorane solution.

2. Remove the bandage and plaster by carefully dissecting these using an artery scissor while avoiding disturbance of the transplant site.

3. Monitor the mice for signs of discomfort (crouching, immobility, alertness, eye discharge and fur changes) and score the transplanted skin for signs of rejection (Figure 5). In case of discomfort or infection, antibiotics can be administered. 


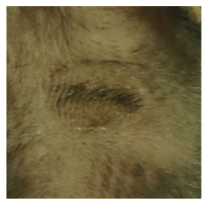

Score 4 : No red areas and presence of a skiny black stripe

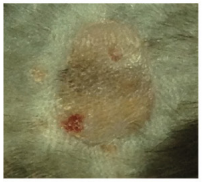

Score 3 : Appearance of small red areas, loss of sheen in black stripe

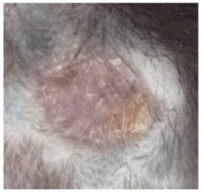

Score 2 : Dryness and complete loss of black stripe

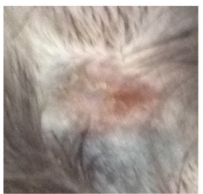

Score 1 : Fibrotic changes, skrinkage or appearance of large red areas

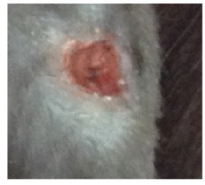

Score 0 : Complete rejection with $>80 \%$ necrosis

Figure 5. Scoring of the skin grafts. The images represent a skin piece of approximately 1 $\mathrm{cm}$ in diameter.

Part II: Lymphoid organ analysis (Lang et al., 2017)

\section{A. Preparation of cells suspensions}

1. Isolation of the lymphoid organs

a. Euthanize the mice with $\mathrm{CO}_{2}$, pin each paw of the mouse using a needle (25 G) to a Styrofoam board (Figure 6A), open the skin in the middle of the abdomen (Figure 6B, arrow) to make an incision through the all body (double $Y$ forms: Figure $6 \mathrm{C}$ ), and detach the skin from the peritoneal membrane. Use needles $(25 \mathrm{G})$ to fix the skin to the styrofoam board (Figure 6D)
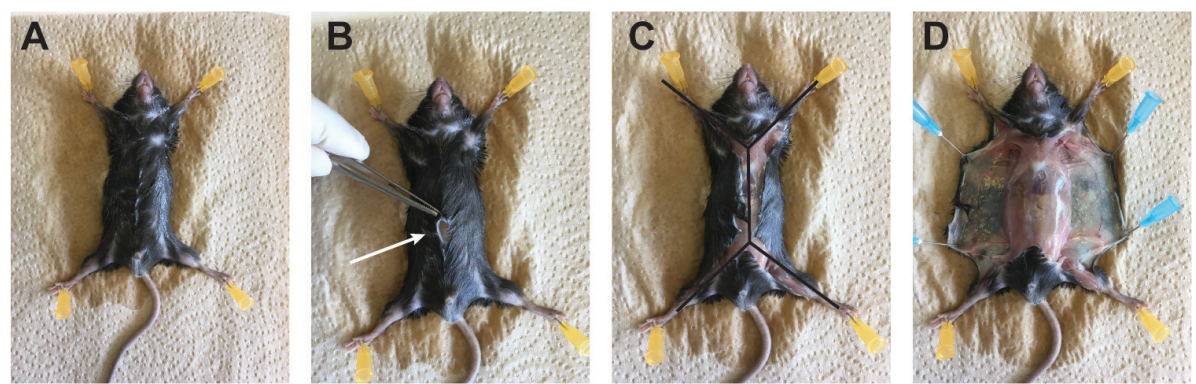

Figure 6. Opening of the mouse skin. A. After pinning the mice on a tray, spray ethanol $70 \%$

on it. B. Make a small skin incision on the 1/3 lower part of the mice. C. Cut the skin throw the upper part of the body and following the 2 arms left and right. Then to the 2 legs left and right. 
Double $Y$ shape. D. Detach the skin from the peritoneal membrane and pin the 2 sides.

b. Take the inguinal and axillary lymph nodes first (Figures 7A-7B), then cut the peritoneal cavity to take the spleen (Figure $7 \mathrm{C}$ ). Open the thoracic cage on the right and left side, leave it up to uncover the heart. Take the thymus on the top of the heart (Figure 7D).

c. Place spleen, thymus, lymph nodes into separate tubes with ice cold PBS.
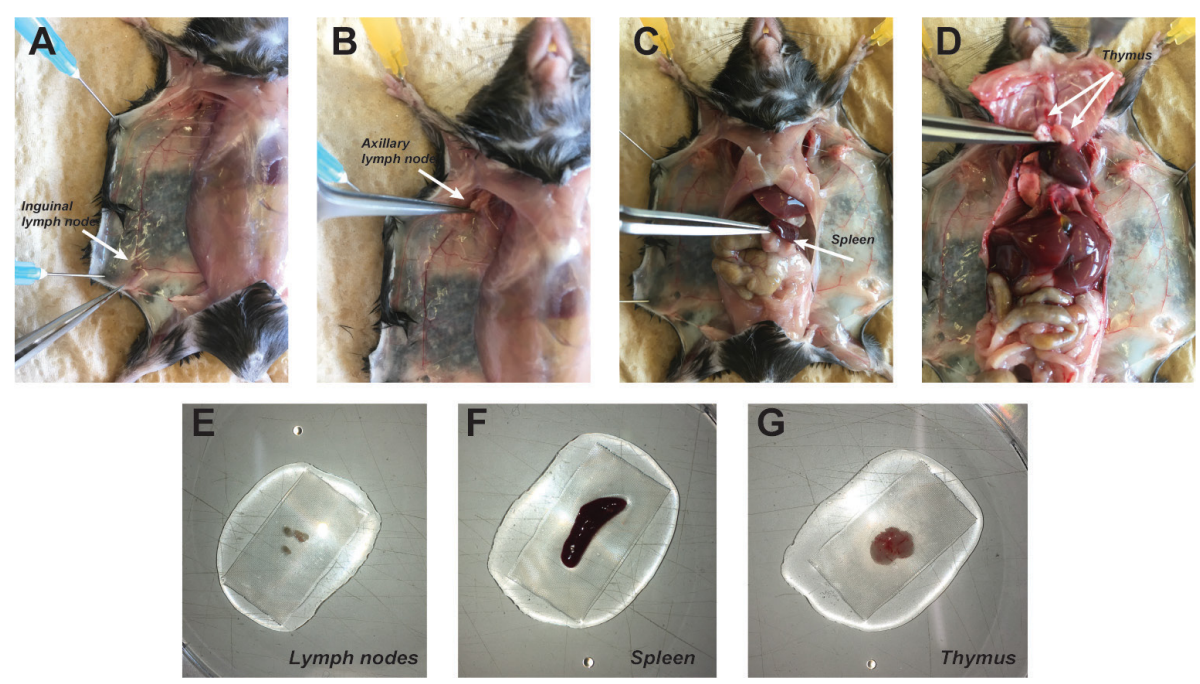

Figure 7. Lymphoid organs harvest. All pointed by the tip of the thin tweezer. A. Remove the inguinal lymph node at the 3 veins intersections (here shown on the left side). B. Remove the axillary lymph nodes below the elbow. C. Spleen removal $D$. Thymus removal, white 2 lobes on the top of the heart. E-G. Three kind of lymphoid organs harvested and placed on grid with PBS in a Petri dish.

2. Cells suspensions of the solid tissues at RT

a. Put $1 \mathrm{ml}$ of PBS on a gridded mesh in a Petri dish, then place the spleen, thymus, lymph nodes from Part II A1 on it (Figure 7E-G)

b. Take the plunger of a $5 \mathrm{ml}$ syringe to smash the organs against the mesh until no solid structure is visible (until the spleen capsule become transparent)

c. Take $1 \mathrm{ml}$ of fresh cold PBS to wash the mesh and the Petri dish, transfer the supernatant containing the cells into a $15 \mathrm{ml}$ tube.

d. Remove the cell debris by a quick spin $(70 \times \mathrm{g}, 5 \mathrm{~s})$. Take the supernatant into a new $15 \mathrm{ml}$ tube. Pellet the cells by $450 \times g, 5$ min spin.

e. Resuspend the cells into the desired volume of PBS for counting $(7 \mathrm{ml}$ for a spleen or a thymus, $1 \mathrm{ml}$ for the lymph nodes).

3. RBC lysis of splenocytes

a. Once the cell debris are removed, pellet the cells by $450 \times \mathrm{g}, 5 \mathrm{~min}$ spin and resuspend them in $1 \mathrm{ml}$ of ACK buffer, let the cell suspension incubate for $1 \mathrm{~min}$ at RT to lyse the RBC. 
b. Add 5 times the volume of ACK ( $5 \mathrm{ml}$ ) of PBS then spin down the cells, $450 \times \mathrm{g}, 5 \mathrm{~min}$.

c. Resuspend the pellet in the desired volume of PBS for counting.

\section{B. Counting}

1. Dilution of the samples in Trypan Blue (1/1).

2. Add $10 \mu \mathrm{l}$ to a Neubauer chamber slide.

3. Determine viable (non-blue) cells in the middle quadrant.

4. Number of cells $=\mathrm{n} \times 2$ (dilution factor) $=2 \mathrm{n} \times 10^{4}$ cells $/ \mathrm{ml}$.

C. Cell surface staining and Flow Cytometry

1. Prepare the FACS antibody mix, one per FACS panel wanted (antibodies should be titrated to obtain optimal results)

2. Plate the cells in the 96 -well plate ( $U$ bottom non treated for culture), $1 \times 10^{6}-2 \times 10^{6}$ cells per well.

3. Centrifuge the cells in the plate by $450 \times g, 5$ min using a rotor equipped with a plate holder.

4. Resuspend the pellet with $50 \mu \mathrm{l}$ of FACS antibodies mix by pipetting up and down.

5. Incubate the plate at $4{ }^{\circ} \mathrm{C}$ for $15 \mathrm{~min}$.

6. Centrifuge the cells in the plate: $450 \times \mathrm{g}, 5 \mathrm{~min}$.

7. Wash the cells in $200 \mu$ l of FACS buffer.

8. Centrifuge the cells using by $450 \times g, 5 \mathrm{~min}, 4^{\circ} \mathrm{C}$.

9. Resuspend the cell pellets in $200 \mu \mathrm{l}$ of FACS buffer, and transfer them in $5 \mathrm{ml}$ FACS tubes for FACS acquisition.

D. Blood assessment and treatment for FACS staining

1. Blood assessment

a. With a $1 \mathrm{ml}$ syringe and $25 \mathrm{G}$ needle target the tip of the ventricle and aspirate $200 \mu \mathrm{l}$ of blood (Figure 8 ) that you transfer in a tube with $40 \mu$ of $2 \%$ EDTA.
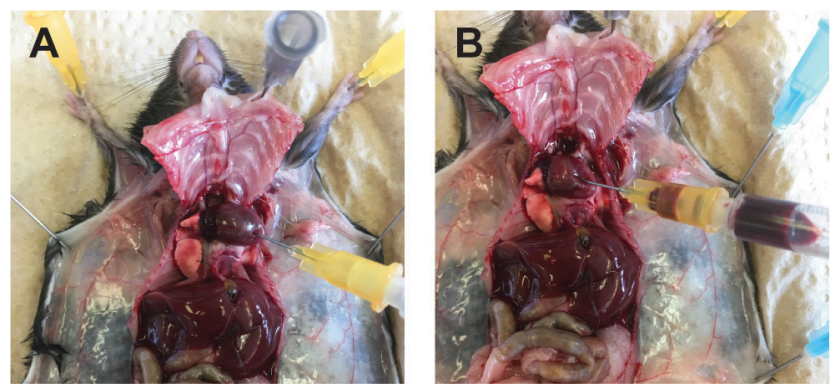

Figure 8. Blood assessment. A. Punch a $25 \mathrm{G}$ needle on the tip of the heart ventricle. B.

Slowly pull out the plunge of the syringe to take the blood.

\section{FACS staining}

a. Transfer $100 \mu \mathrm{l}$ of the blood/EDTA mixture to a $5 \mathrm{ml}$ FACS tube. 
b. Add $50 \mu$ of FACS buffer containing the correct amount of antibodies (Table 1).

c. Vortex briefly (speed 8).

d. Incubate for $20 \mathrm{~min}$ at $4{ }^{\circ} \mathrm{C}$ (in the dark to avoid photobleaching).

e. Add $3 \mathrm{ml}$ of FACS buffer.

f. Centrifuge cells for $5 \mathrm{~min}$ at $450 \times \mathrm{g}, 4^{\circ} \mathrm{C}$.

g. Aspirate the supernatant using a yellow tip (P200) connected to a vacuum pump

Table 1. FACS panel example

\begin{tabular}{ll}
\hline Antigen & Fluorophore \\
\hline CD3 & AF488 \\
CD4 & Pacific Orange \\
CD8 & Pacific Blue \\
CD62L & PE \\
Gr1 & APC \\
CD11b & PE/Cy7 \\
B220 & PerCP \\
Live/Dead marker & APC/Cy7 \\
\hline
\end{tabular}

3. RBC lysis

a. Add $1 \mathrm{ml}$ of $1 \mathrm{x}$ FACS lysing solution to the blood (will be turbid at this stage, see Figure 9A), vortex briefly (speed 8).

b. Wait until the solution becomes clear (transparent; should take about $1 \mathrm{~min}$ ) (Figure 9B).

c. Add $3 \mathrm{ml}$ of FACS buffer.

d. Centrifuge for $5 \mathrm{~min}$ at $450 \times \mathrm{g}, 4^{\circ} \mathrm{C}$.

e. Decant the supernatant.

f. Resuspend the pellets in $250 \mu$ of FACS buffer for FACS acquisition.

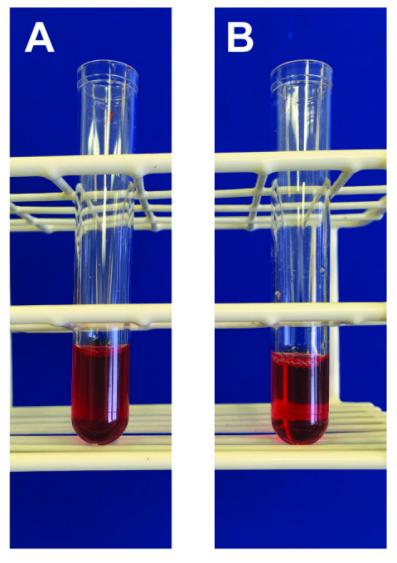

Figure 9. Blood lysis. A. Blood prior to RBC lysis. B. Blood after RBC lysis. 


\section{E. FACS acquisition and analysis}

We do the FACS acquisition on a BD FACS Canto or BD LSR Fortessa with Diva software and analyzed using FlowJo Software. Example of a FACS staining for thymus (Figure 10).
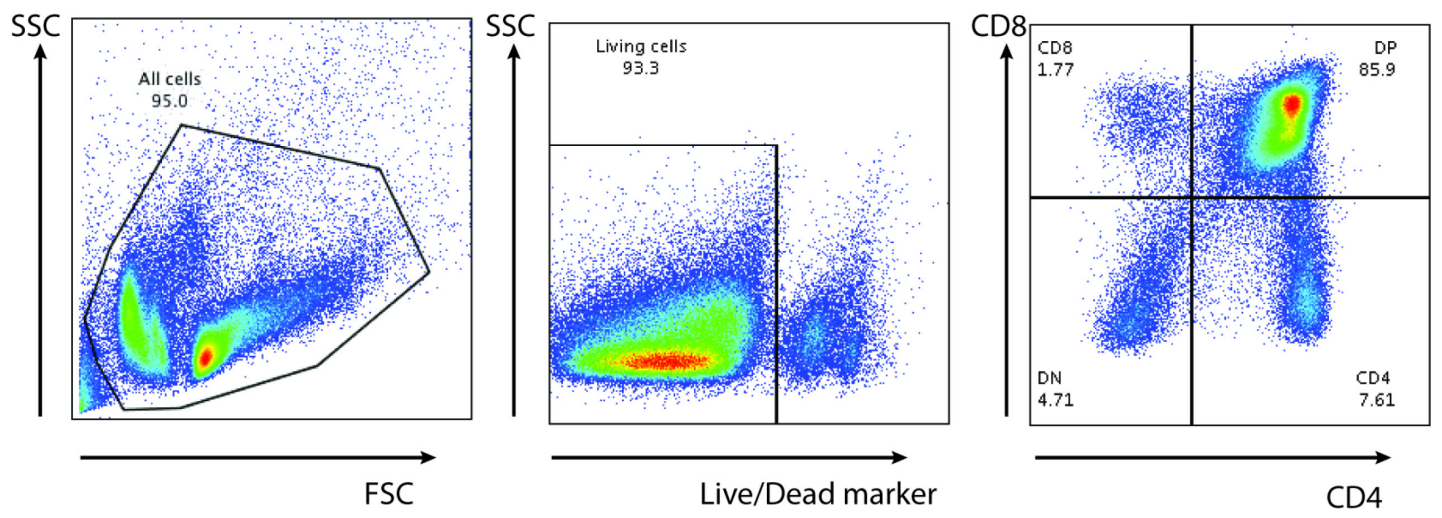

Figure 10. Thymocytes stained for $T$ cells

\section{Acknowledgments}

Work in the Pieters Laboratory is supported by the Swiss National Science Foundation, the Swiss Multiple Sclerosis Society, the Novartis Foundation for Medical-Biological Research, the Gebert Ruef Foundation, and the Canton of Basel. Rajesh Jayachandran was supported by a grant from the Prof. Max Cloetta Foundation. The Rossi laboratory has been supported by the Swiss National Foundation and the Freiwillige Akademische Gesellschaft (FAG) Basel. The procedure used here has been adapted and modified from Siegmund et al., 2011; Schmaler et al., 2014; Lang et al., 2017; Jayachandran et al., 2019.

\section{Competing interests}

The authors declare no conflict of interest.

\section{Ethics}

In this protocol all animal procedures were conducted in accordance to the animal protocol approved by the Cantonal Authority Basel-Stadt, Switzerland, Animal licence 1893 (01.01.2017-01.01.2020).

\section{References}

1. Green, M. (2013). Introduction: Infections in solid organ transplantation. Am J Transplant 13 Suppl 4: 3-8.

2. Jayachandran, R., Gumienny, A., Bolinger, B., Ruehl, S., Lang, M. J., Fucile, G., Mazumder, S., 
Tchang, V., Woischnig, A. K., Stiess, M., Kunz, G., Claudi, B., Schmaler, M., Siegmund, K., Li, J., Dertschnig, S., Hollander, G., Medina, E., Karrer, U., Moshous, D., Bumann, D., Khanna, N., Rossi, S. W. and Pieters, J. (2019). Disruption of coronin 1 signaling in T cells promotes allograft tolerance while maintaining anti-pathogen immunity. Immunity 50(1): 152-165.e158.

3. Lang, M. J., Mori, M., Ruer-Laventie, J. and Pieters, J. (2017). A coronin 1-dependent decision switch in juvenile mice determines the population of the peripheral naive T cell compartment. $J$ Immunol 199(7): 2421-2431.

4. Lopez, M. M., Valenzuela, J. E., Alvarez, F. C., Lopez-Alvarez, M. R., Cecilia, G. S. and Paricio, P. P. (2006). Long-term problems related to immunosuppression. Transpl Immunol 17(1): 3135.

5. McKenzie, I. F., Morgan, G. M., Sandrin, M. S., Michaelides, M. M., Melvold, R. W. and Kohn, H. I. (1979). B6.C-H-2bm12. A new H-2 mutation in the I region in the mouse. J Exp Med 150(6): 1323-1338.

6. Rodriguez-Peralvarez, M., De la Mata, M. and Burroughs, A. K. (2014). Liver transplantation: immunosuppression and oncology. Curr Opin Organ Transplant 19(3): 253-260.

7. Schmaler, M., Broggi, M. A. and Rossi, S. W. (2014). Transplantation of tail skin to study allogeneic CD4 T cell responses in mice. J Vis Exp(89): e51724.

8. Siegmund, K., T. Zeis, G. Kunz, T. Rolink, N. Schaeren-Wiemers and J. Pieters (2011). Coronin 1-mediated naive $T$ cell survival is essential for the development of autoimmune encephalomyelitis. J Immunology 186(6): 3452-3461. 